\title{
Performance of Jet and Missing Transverse Energy Reconstruction with CMS in pp Collisions at $\sqrt{s}=7$ TeV
}

Joanna Weng*on behalf of the CMS collaboration

Author affiliation: Institute for Particle Physics, ETH Zurich, 8093 Zurich, Switzerland

E-mail: Joanna.Weng@cern.ch

We present the techniques adopted by CMS to measure the jet energy response and resolutions as well as the Missing Transverse Energy. Results from detailed Monte Carlo studies are compared with preliminary observations from the collider data based on up to $73 \mathrm{nb}^{-1}$ of proton-proton LHC collisions at $7 \mathrm{TeV}$ center of mass energy. Studies for jet energy response and resolutions are presented for three different approaches to reconstruct jets in the CMS detector: calorimeteronly based jet reconstruction, the "Jet-Plus-Track" and the "Particle Flow" method.

The studies of the Missing Transverse Energy $\left(\mathbb{E}_{T}\right)$ reconstruction are based on a data sample of up to $11.7 \mathrm{nb}^{-1}$ of integrated luminosity. Three missing transverse energy reconstruction algorithms, corresponding to the jet reconstruction approaches, are studied: pure calorimeter $\mathbb{E}_{T}$, calorimeter $\mathbb{E}_{T}$ corrected with track momenta and $\mathbb{E}_{T}$ calculated using the "Particle Flow" technique. We discuss the cleaning and the performance of these three reconstruction methods using minimum-bias data and data selected to contain high transverse momentum jets.

35th International Conference of High Energy Physics - ICHEP2010,

July 22-28, 2010

Paris France

${ }^{*}$ Speaker. 


\section{Jet Performance}

Four types of jets are reconstructed at CMS, which differently combine individual contributions from sub detectors to form the inputs to the jet clustering algorithm: Calorimeter jets are reconstructed using energy deposits in the electromagnetic (ECAL) and hadronic (HCAL) calorimeter cells, combined into calorimeter towers. The Jet-Plus-Tracks (JPT) algorithm [1] exploits the excellent performance of the CMS tracking detectors to improve the $p_{\mathrm{T}}$ response and resolution of calorimeter jets. Starting from a calorimeter jet, the momenta of the tracks associated to the jet are added and the expected average energy deposition in the calorimeters is subtracted. The Particle Flow (PFlow or PF) algorithm [2, 3] combines the information from all CMS sub-detectors to identify and reconstruct all particles in the event, namely muons, electrons, photons, charged hadrons and neutral hadrons. Track jets are reconstructed from well measured tracks [4]. The clustering for all types is performed with the anti- $k_{T}$ algorithm [5] with jet size parameter $R=0.5$. The jet results are based on data up to $73 \mathrm{nb}^{-1}$ of proton-proton collisions recorded with the CMS detector [6].

CMS has developed a factorized multi-step procedure for the jet energy calibration back to the particle jet level (JEC) [7]. The two most important corrections, the absolute and the relative correction, are discussed in the following.

\subsection{Relative response from dijet $p_{\mathrm{T}}$ balance}

The relative correction remove variations in jet response versus jet $\eta$ relative to a central control region $(|\eta|<1.3)$ chosen as a reference because of the uniformity of the detector. It is based on $p_{\mathrm{T}}$ balance in dijet events and has been used to measure the jet response as a function of pseudorapidity.
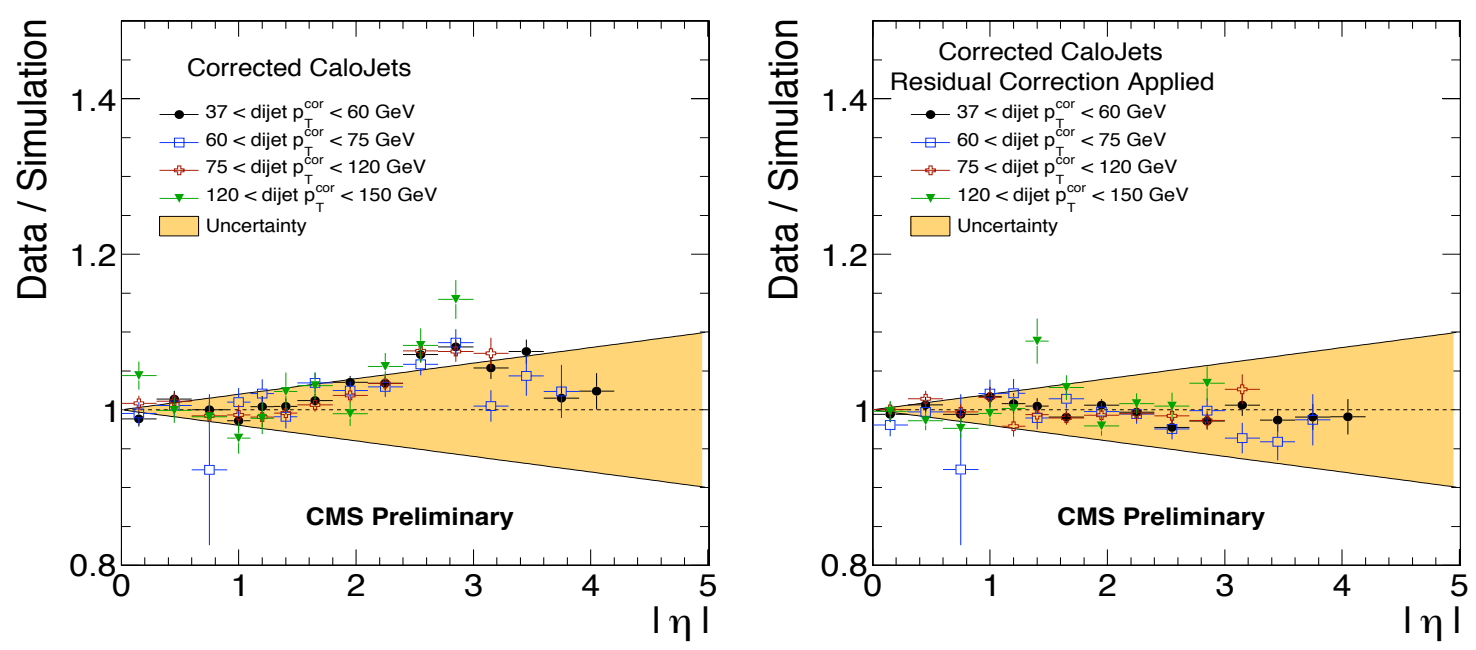

Figure 1: Left: Data/MC ratio for the relative response obtained from the dijet $p_{\mathrm{T}}$ balance method for calorimeter jets. The $\pm 2 \% \times|\eta|$ uncertainty band is overlaid. Right: Same data/MC ratio for the relative response, but this time jets in data are corrected in addition with the residual correction discussed in the text.

Figure 1 (left) shows the data/MC (PYTHIA 6) ratio for the relative response, as measured with the dijet balance method. In order to account for the observed shift in the data, we derived 

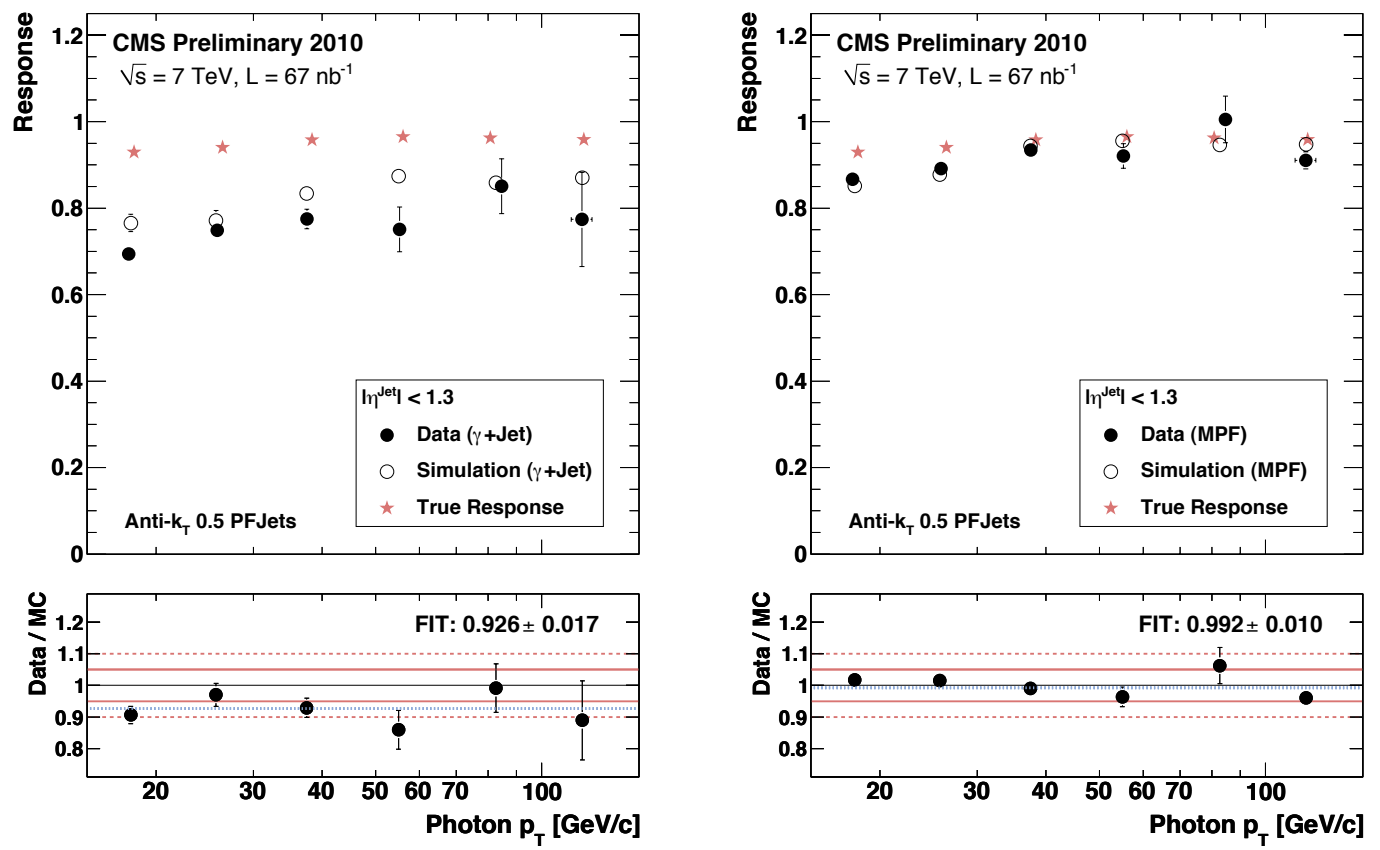

Figure 2: Response $<p_{\mathrm{T}} / p_{\mathrm{T}}^{\gamma}>$ versus $p_{\mathrm{T}}^{\gamma}$ in data and MC (left) and MPF response in data and MC (right) for $\mathrm{PF}$ jets. The MC truth response is also shown. The data/MC ratio and the one-parameter linear fit function is shown at the bottom of the plots, together with $\pm 5 \%$ and $\pm 10 \%$ lines.

an additional residual data driven correction. Figure 1 (right) shows the same data/MC ratio after jets in data are additionally corrected using the residual correction. We assign an uncertainty of $\pm 2 \% \times|\eta|$ which after the residual corrections is clearly a conservative estimate, as can be seen in Figure 1.

\subsection{Absolute response measurements from photon+jet events}

The absolute correction removes variations in jet response versus jet $p_{\mathrm{T}}$. The $p_{\mathrm{T}}$ balance and the MPF (missing $E_{T}$ projection fraction) methods in photon+jet events have been used to study the jet response in the central region as a function of jet $p_{\mathrm{T}}$. Both methods exploit the balance in the transverse plane between the photon and the leading jet and use the photon $p_{\mathrm{T}}$, that is accurately measured in the crystal ECAL calorimeter, as a good estimator of the parton level jet $p_{T}$.

As can be seen in Figure 2 (left), the jet response extracted from the $\gamma+$ jet balancing exhibits sizeable negative bias, compared to the true jet response. This effect is currently expected with the limited amount of data and is attributed to the presence of additional jets in the event which spoil the idealized topology of a jet recoiling against a photon.

Therefore, we also use the MPF method which starts from the simple assumption that the $\gamma+$ jet events have no intrinsic missing $E_{T}$ and that the photon is perfectly balanced by the hadronic recoil in the transverse plane. Distributions of MPF response in the selected $\gamma+$ jet calibration sample are shown in Figure 2 (right) for the same representative $p_{\mathrm{T}}^{\gamma}$ bins as before. It can be seen that the MPF response agrees better with the true response than $p_{\mathrm{T}}$ balancing response does, because it 
is less sensitive to the presence of secondary jets in the event. Observed agreement between data and MC when the same MPF method is applied indicates that the simulation describes the detector response within $5 \%$ precision.

\subsection{Jet Energy resolutions}

Jet energy resolutions have been studied using the asymmetry method in dijet data events. Events are required to contain at least two jets within $|\eta|<1.4$, with the leading jet pair being azimuthally separated by $\Delta \phi>2.7$. For approximately equal $p_{\mathrm{T}}$ values of both jets, and their resolutions, assuming also the same resolution, the variance $\sigma$ of the asymmetry distribution $A=$ $\frac{p_{\mathrm{T}}^{\text {jett }}-p_{\mathrm{T}}^{\text {jert }}}{p_{\mathrm{T}}^{\text {jetl }}+p_{\mathrm{T}}^{\text {jer2 }}}$ can be related to the jet $p_{T}$ resolution by $\frac{\sigma\left(p_{\mathrm{T}}\right)}{p_{\mathrm{T}}}=\sqrt{2} \sigma_{A}$.

the variance of the asymmetry $\sigma$ can be related to the jet $p_{\mathrm{T}}$ resolution. The full dijet asymmetry analysis procedure is applied to the selected data sample and compared to the results from a PYTHIA 6 QCD dijet sample obtained with the same method.

While still limited by the size of the available data sample, data and simulation are in reasonable agreement, typically within $10 \%$. As expected, the resolutions for jets employing tracking information are better compared to the calorimeter jets. More details about CMS jet performance can be found in $[8,9]$.

\section{Missing Transverse Energy Performance $\left(\mathbb{t}_{T}\right)$ :}

The goal at this early stage is to understand the performance of the $\mathbb{E}_{T}$ measurement using events where there is no significant intrinsic $\mathbb{E}_{T}$. CMS has implemented three main types of algorithms to reconstruct Missing Transverse Energy: (i) $\mathbb{E}_{T}$ based on calorimeter energies using the tower geometry of the hadron calorimeter (calo $\mathbb{E}_{T}$ ), (ii) $\mathbb{E}_{T}$ calculated by replacing the calorimeter tower energies matched to charged hadrons with their corresponding charged-track momenta (track-corrected $\mathbb{E}_{T}$ ) (iii) $\mathbb{E}_{T}$ calculated from particles reconstructed with the PF algorithm (PF $\mathbb{E}_{T}$ ) In the following we present first results of the three $\mathbb{E}_{T}$ reconstruction approaches using up to $11.7 \mathrm{nb}^{-1}$ of data. More details can be found in $[9,10]$.

\section{Missing Transverse Energy Cleaning}

We studied the effect of instrumental anomalies and beam-induced backgrounds on the $\mathbb{E}_{T}$ measurement and demonstrated a cleaning procedure to identify and correct for these effects. The basic strategy is to identify anomalous signals based on non-physical charge sharing between neighboring channels in space and/or depth, as well as timing / pulse shape information. The $\mathbb{E}_{T}$ distributions after different stages of the anomalous signal removal procedure in the minimum-bias data sample are shown in Figure 3 (left), demonstrating the effectiveness of the anomalous signal removal.

\section{Missing Transverse Energy Resolution}

We compare the $\mathbb{E}_{T}$ resolution as a function of $\sum E_{T}$ for the three $\mathbb{E}_{T}$ reconstruction techniques described above with each other and with the Monte Carlo simulation. We characterize the $\mathbb{E}_{T}$ resolution based on the $\sigma$ of a Gaussian fit to the missing $E_{x, y}$ distribution. In order to make a 

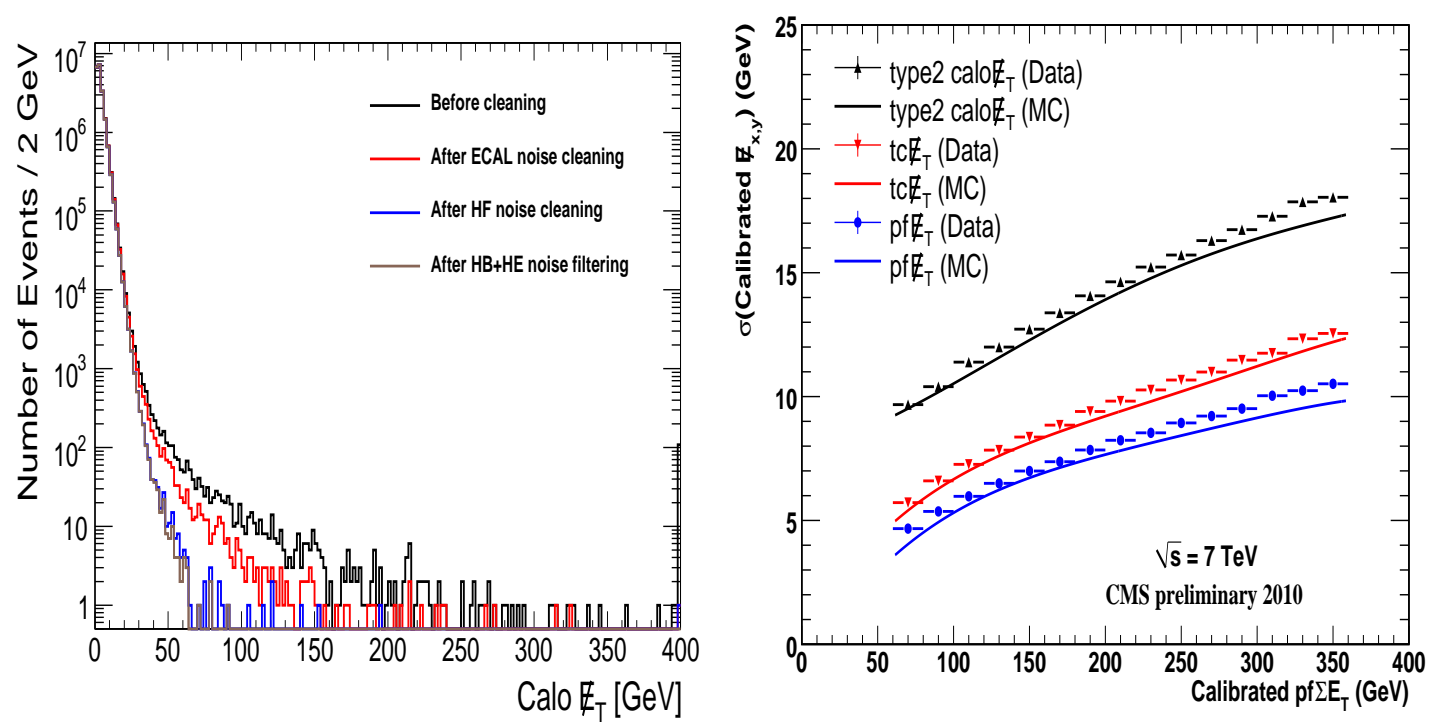

Figure 3: Left: Calo $\mathbb{E}_{T}$ distributions after different stages of the anomalous signal removal procedure. Right: Calibrated $\mathbb{E}_{T}$ resolution versus calibrated pf $\Sigma E_{\mathrm{T}}$ for the type-II corrected calo $\mathbb{E}_{T}$, track-corrected $\mathbb{E}_{T}$, and PF $\mathbb{E}_{T}$ in data and Monte Carlo samples.

meaningful comparison, we calibrate the measured $\mathbb{E}_{T}$ to be in agreement with the true $\mathbb{E}_{T}$. The calibration factors are extracted from Monte Carlo (PYTHIA 8) using photon+jets events. From the calibrated $\mathbb{E}_{T}$, new missing $E_{x, y}$ values are calculated and the $\mathbb{E}_{T}$ resolution is obtained.

For $\sum E_{T}$, we use the PF $\sum E_{T}$ as measured by the particle-flow algorithm for all types of $\boldsymbol{E}_{T}$. We calibrate PF $\Sigma E_{\mathrm{T}}$ to the particle-level $\sum E_{T}$, on average, based on the correlation between the measured PF $\Sigma E_{\mathrm{T}}$ and the particle-level $\Sigma E_{\mathrm{T}}$ observed in MC. Figure 3 (right) shows the calibrated $\mathbb{E}_{T}$ Gaussian core resolution versus the calibrated PF $\Sigma E_{\mathrm{T}}$ for different $\mathbb{E}_{T}$ reconstruction algorithms in events containning at least two jets with $p_{T}>25 \mathrm{GeV}$. Both track-corrected $\mathbb{E}_{T}$ and, especially, PF $\mathbb{E}_{T}$ improve the resolution compared to the purely calorimetric $\mathbb{E}_{T}$ reconstruction. The data/MC agreement is acceptable for this early stage of $\mathbb{E}_{T}$ commissionning.

\section{Conclusions}

We have presented the first measurements of jet energy calibration and jet resolutions as well as the reconstruction of $\mathbb{E}_{T}$ in early pp collisions recorded by CMS at $\sqrt{s}=7 \mathrm{TeV}$. Significantly better performance for the objects employing the tracking information have been observed compared to the objects using calorimeter-only information. The results in the jet sector support our estimates of $10 \%$ (5\%) jet calibration uncertainties for calorimeter jets (Jet-Plus-Track and Particle Flow jets), with the additional $2 \%$ uncertainty per unit rapidity as conservative estimates.

After the cleaning procedure, the $\mathbb{E}_{T}$ and $\Sigma E_{\mathrm{T}}$ distributions, as well as the $\mathbb{E}_{T}$ resolutions in the data are generally in good agreement with the Monte Carlo simulation predictions, although still some observed differences need to be investigated in future studies. 


\section{References}

[1] CMS Collaboration, Jet Plus Tracks Algorithm for Calorimeter Jet Energy Corrections in CMS, CMS PAS JME-09-002 (2009).

[2] CMS Collaboration, Commissioning of the Particle-flow Event Reconstruction with the first LHC Collisions recorded in the CMS detector, CMS PAS PF-10-001 (2010).

[3] CMS Collaboration, Commissioning of the Particle-Flow reconstruction in Minimum-Bias and Jet Events from pp Collisions at $\sqrt{s}=7$ TeV, CMS PAS PF-10-002 (2010).

[4] CMS Collaboration, Commissioning of Track Jets in pp Collisions at $\sqrt{s}=7 \mathrm{TeV}$, CMS PAS JME-10-006 (2010).

[5] Cacciari, M. and Salam, G. P. and Soyez, G., The anti-kt jet clustering algorithm, JHEP 0804:063 (2008).

[6] CMS Collaboration, The CMS experiment at the CERN LHC, JINST 3 S08004 (2008).

[7] CMS Collaboration, Plans for Jet Energy Corrections at CMS, CMS PAS JME-07-002 (2008).

[8] CMS Collaboration, CMS Jet Performance in pp Collisions at $\sqrt{s}=7 \mathrm{TeV}$, CMS PAS JME-10-003 (2010).

[9] CMS Collaboration, Jet and MET Commissioning Results from 7 TeV Collision Data, CMS DP-2010/014 (2010).

[10] CMS Collaboration, Missing Transverse Energy Performance in Minimum-Bias and Jet Events from Proton-Proton Collisions at $\sqrt{s}=7$ TeV, CMS PAS JME-10-004 (2010). 